\title{
Triceps brachii tendon injury in four Pomeranians
}

\author{
Ryosuke ECHIGO ${ }^{1) *}$, Atsushi FUJITA ${ }^{2)}$, Ryohei NISHIMURA ${ }^{3)}$ and \\ Manabu MOCHIZUKI ${ }^{4)}$ \\ ${ }^{1)}$ Veterinary Teaching Hospital, Graduate School of Veterinary Medicine, Hokkaido University, Kita 19 jo Nishi 10 \\ chome, Kita-ku, Sapporo-shi, Hokkaido 060-0818, Japan \\ ${ }^{2)}$ Veterinary Medical Center, Graduate School of Agricultural and Life Sciences, The University of Tokyo, \\ 1-1-1 Yayoi, Bunkyo-ku, Tokyo 113-8657, Japan \\ 3) Laboratory of Veterinary Surgery, Graduate School of Agricultural and Life Sciences, The University of Tokyo, \\ 1-1-1 Yayoi, Bunkyo-ku, Tokyo 113-8657, Japan \\ 4)Laboratory of Veterinary Emergency Medicine, Graduate School of Agricultural and Life Sciences, The University \\ of Tokyo, 1-1-1 Yayoi, Bunkyo-ku, Tokyo 113-8657, Japan
}

J. Vet. Med. Sci.

80(5): 772-777, 2018

doi: $10.1292 /$ jvms.17-0318

Received: 12 June 2017

Accepted: 13 March 2018

Published online in J-STAGE:

22 March 2018
ABSTRACT. The cases of 4 Pomeranians with injury of the triceps brachii tendon that underwent surgical treatment were retrospectively reviewed to evaluate some clinical findings including signalment, cause of injury, clinical signs, pattern of injury, surgical technique, external coaptation after operation, complications, and outcomes. While all of the dogs showed non-weight bearing posture of the affected limbs and severe pain shortly after injury onset, the pain level decreased over time. A characteristic finding of the 4 cases was an absence of tension in the triceps brachii tendon when the elbow joint was flexed. The pattern of triceps brachii tendon injury was either laceration of the central part of the tendon $(n=1)$ or tendon rupture at its insertion to the olecranon $(n=3)$. Although there were no major complications after surgery in 3 cases, the remaining case required a revision surgery. Long lateral splint was effective method for external coaptation after operation. Diagnosis of triceps brachii tendon injury was not difficult if we even recognize this trauma. This form of injury can have a good prognosis with adequate surgery and postoperative coaptation.

KEY WORDS: dog, tendon, three-loop pulley, triceps

In dogs, tendon injuries are typically caused by trauma such as traffic accidents and falls from a height [10, 14, 17]. Tendon conditions can be divided into many patterns (laceration at musculotendinous junction, laceration of the central part of the tendon, rupture at its insertion to the bone, avulsion fracture, contusion, strain) [14, 17]. When severe damage to tendon occurs, the joint related to the tendon is unable to extend or flex by loss of bone movement, resulting in lameness. In the majority of cases, tendon injury can be diagnosed by palpation of the related muscle or joint motion. In cases of avulsed tendon with bone fragment, the fragment can be detected by radiography [6].

Tendon injury can be treated with either conservative therapy or surgical intervention. Because it is rare for the injured tendon to heal with conservative therapy including coaptation and restriction of motion alone, surgical intervention is required in many cases $[9,18]$. The most common method used for apposing tendon ends is suturing each end of the lacerated tendon $[14,16]$. A variety of suturing methods have been investigated including locking loop, far-near near-far, Bunnel-Mayer technique, and the three-loop pulley [14]. In cases of tendon avulsion from the bone, suturing tendon to bone can be achieved by using the modified three-loop pulley $[10,11,15]$. Postoperative immobilization is strongly recommended to prevent failure of the primary repair and to improve outcomes by reducing the incidence of suture pullout or breakage. After surgery, coaptation such as casting and splinting should be performed for 6 to 10 weeks [12]. If appropriate surgery and postoperative management is completed, the outcome following tendon injury is generally excellent $[16,17]$.

The largest muscle in the forelimb is the triceps brachii muscle. In many mammals, this muscle comprises 3 heads (long, lateral, and medial heads); however, an accessory head is also present in dogs [7]. The triceps brachii muscle begins at the caudal border of scapula and proximal humerus, and ends at the olecranon; its main functions include flexion of the scapular joint and extension of the elbow joint. When severe damage to the triceps brachii muscle occurs, the elbow joint continues to flex because of the loss of its extension mechanism, resulting in lameness of the affected limb. 
Table 1. Signalment and clinical data at presentation for 4 dogs with injured triceps brachii tendon

\begin{tabular}{ccccccc}
\hline Dogs & Breed & Age (year) & Sex & BW $(\mathrm{kg})$ & Cause of injury & Duration to presentation (days) \\
\hline No. 1 & Pomeranian & 10 & CM & 3.1 & Jumping from a height & 37 \\
No. 2 & Pomeranian & 2 & M & 2.9 & Jumping from a height & 30 \\
No. 3 & Pomeranian & 9 & SF & 4.7 & Unknown & 35 \\
No. 4 & Pomeranian & 2 & SF & 5.0 & Jumping from a height & 90 \\
\hline
\end{tabular}

BW: Body weight, CM: Castrated male, M: Male, SF: Spayed female.

While there are some reports describing injured triceps brachii tendon, most of them are a single case reports $[2,3,5,13,20]$. The purpose of this study is to examine the clinical characteristics, treatment, and outcomes of multiple cases of triceps brachii tendon injury in dogs. To the best of our knowledge, this is the first report to describe multiple cases of triceps brachii tendon injury.

\section{MATERIALS AND METHODS}

Medical records (April 2007-March 2013) were reviewed to identify dogs with injury of the triceps brachii tendon at Veterinary Medical Center (VMC) of the University of Tokyo; all cases were referred by a private veterinary hospital. Data collected from the medical records included signalment (breed, sex, age, and body weight), cause of injury, clinical signs, pattern of injury, surgical technique, external coaptation after operation, complications, and outcomes. Outcome was determined by follow-up at least 6 months postsurgically by either physical examination or information derived from telephone conversations with the dog owner. This study was explained to the owners and informed consent was obtained from them.

\section{RESULTS}

\section{Signalment, cause of injury}

Total of 4,777 number cases were reviewed, 5 cases were identified to have injury of triceps brachii tendon. Four out of 5 cases were Pomeranians, and the other one is Shetland sheepdog. The odds ratio of Pomeranians was 122.5 (95\% CI: 13.6-1102.2). For a case of Shetland sheepdog which had severe tendon injury with bone fragment due to extreme trauma, it was excluded from the subsequent study because the condition was too different from the other 4 cases. The median of age at diagnosis was 5.5 (range: 2-10) years and the median of body weight was 3.9 (range: $2.9-5.0 \mathrm{~kg}$ ) (Table 1). There were 1 intact male, 1 neutered male, and 2 spayed females. The cause of injury was not known in 1 dog but clearly established in the other 3 . In the 3 dogs, injury was the result of a hard landing caused by jumping from a height (Table 1).

\section{Clinical signs from the onset to referral}

All 4 dogs showed non-weight bearing posture of the affected limb and severe pain shortly after injury. Pain was detected by palpating the caudal part of distal brachium. Pain level decreased over time and eventually disappeared; however, gait did not improve. Non-weight bearing lameness of the affected limb was sustained continuously, despite the application of conservative therapy (administration of some kinds of nonsteroidal anti-inflammatory drugs, external Robert-Jones bandage, and restriction of motion) before arrival at VMC.

\section{Diagnosis}

The dogs were referred to the VMC 30 to 90 days after injury onset. At the first visit to VMC, all of the dogs were in excellent physical condition except for lameness of the affected limb. All blood examination values, including complete blood count and biochemical analysis (alanine aminotransferase, alkaline phosphatase, blood urea nitrogen, creatinine, and C-reactive protein) were in the normal range. In the standing position, all dogs held up the affected limb with the elbow flexed (Fig. 1). On palpation, the affected limbs, in particular the brachium, were thinner than the contralateral limbs due to diffuse muscle atrophy. Nodular lesions were palpated around the triceps brachii tendon near the olecranon. When the elbow joint was flexed, there was no detection of tension in the triceps brachii. Pain was not present when palpating the brachium or when moving the elbow joint. Although there were no significant radiological findings from the anterior-posterior view, continuity of the triceps brachii disappeared from the mediolateral view (Fig. 2).

\section{Perioperative anesthesia}

Dogs were premedicated with atropine $(0.05 \mathrm{mg} / \mathrm{kg}$ intravenously [IV]) followed by anesthesia, which was induced using IV propofol and maintained with isoflurane in oxygen. An appropriate dose of fentanyl was administered as a continuous IV infusion in the perioperative period, and cefazolin $(20 \mathrm{mg} / \mathrm{kg} \mathrm{IV})$ was administered at induction and every $2 \mathrm{hr}$ during surgery.

\section{Surgical findings and technique}

Dogs were positioned in lateral recumbent position, with the affected limb being uppermost. The skin incision was initiated 


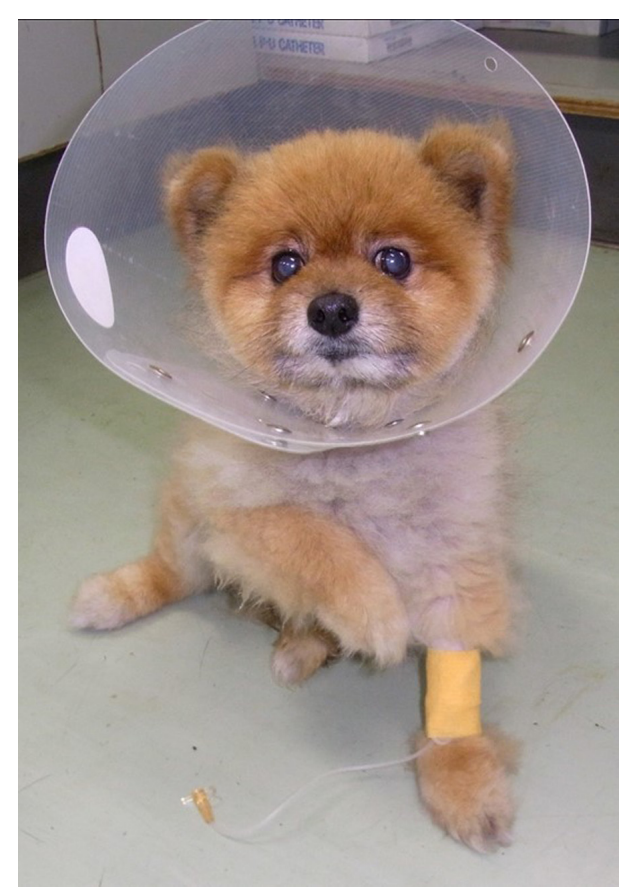

Fig. 1. The posture to flex the elbow joint and hold the affected right forelimb up in Dog 3.
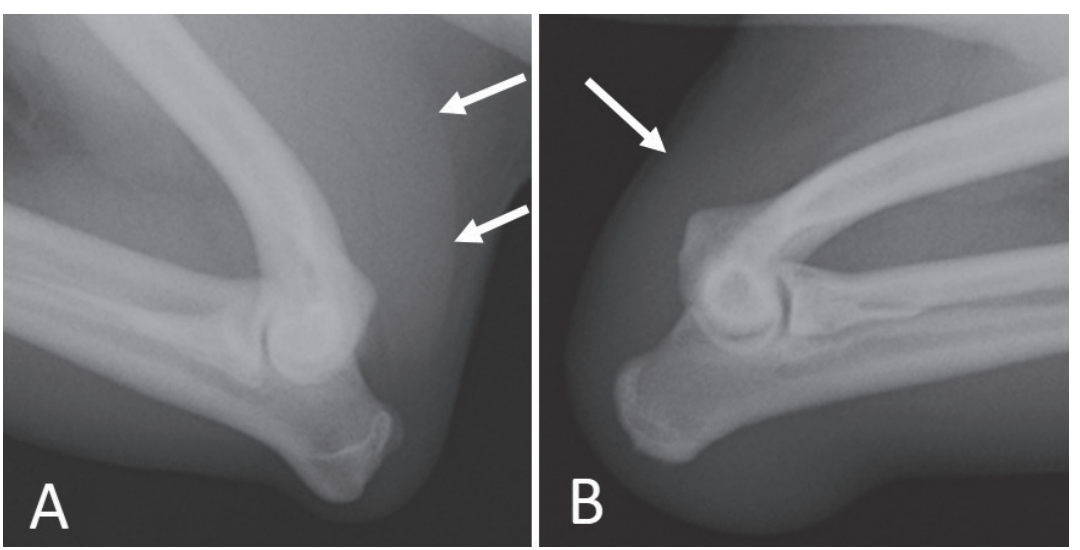

Fig. 2. Radiographic images of the both elbow joints in Dog 2. (A) Mediolateral view of the intact right elbow; note that soft tissue indicating the triceps brachii is clearly observable (arrows). (B) Mediolateral view of the injured left elbow; note the disappearance of the continuity of the triceps brachii (arrow).
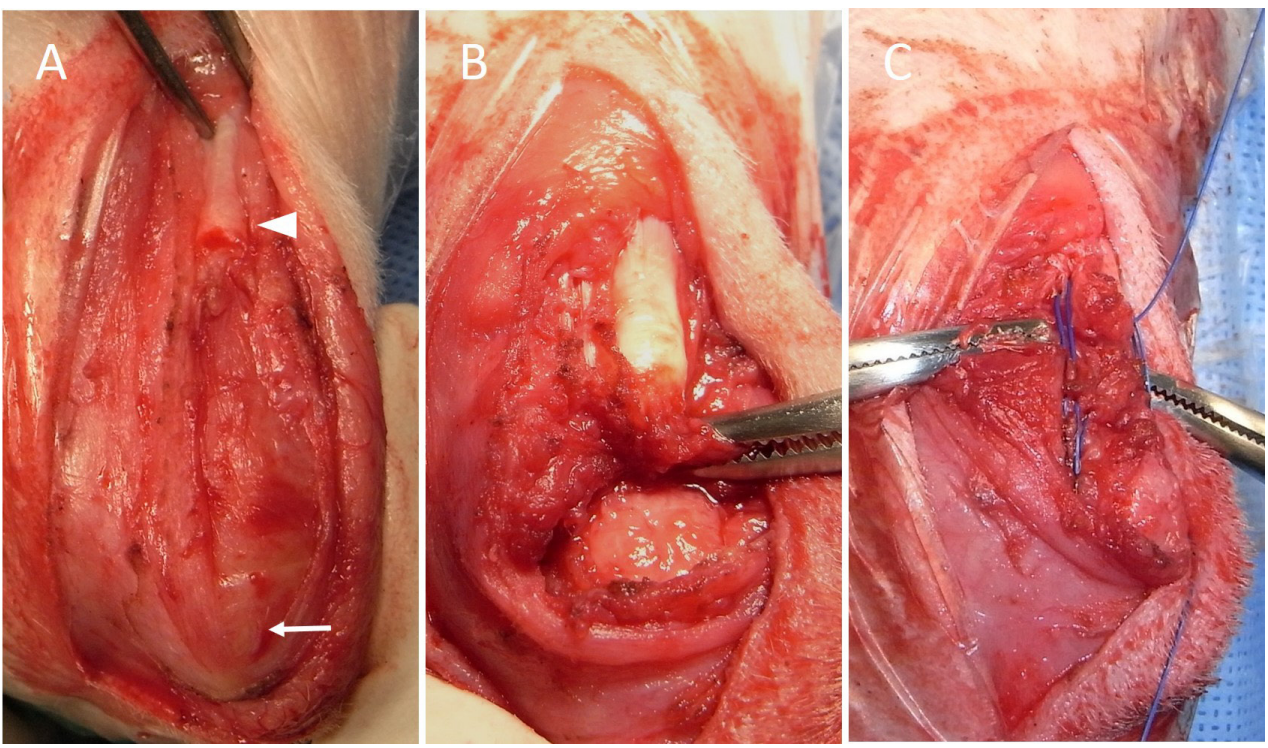

Fig. 3. The injured triceps brachii tendon in Dog 2. (A) On flexion of the elbow joint, the proximal edge of the triceps tendon (arrowhead) was far apart from the olecranon (arrow). (B) After trimming the surrounding soft tissue, the distance between the edge and the olecranon decreased when the elbow joint extended. (C) The modified three-loop pulley was applied to reduce tendon avulsion; this suture method was applied between the proximal edge of tendon and the olecranon.

approximately distal and caudal to the midshaft of the humerus, and continued over the olecranon. Subcutaneous fat and fascia were incised on the same line as the skin and retracted laterally and medially. Following this, the triceps brachii muscle and its tendon were investigated in detail (Fig. 3A). While the central part of triceps brachii tendon was lacerated in only 1 of the cases (No. 1), the type of tendon rupture at its insertion to the olecranon was observed in the other 3 cases (Nos. 2-4) (Table 2). In the case with the laceration type (No. 1), both edges of lacerated tendon formed nodular lesions and were trimmed sharply using a scalpel. Both edges were brought close to each other by extending the elbow joint and sutured according to the three-loop pulley 
Table 2. Clinical finding, treatment, and outcome for 4 dogs with injured triceps brachii tendon

\begin{tabular}{|c|c|c|c|c|c|c|}
\hline Dogs & Pattern of tendon injury & Surgical procedure & Type of coaptation & $\begin{array}{c}\text { Duration of } \\
\text { coaptation (days) }\end{array}$ & Complication & $\begin{array}{c}\text { Duration of } \\
\text { follow-up (days) }\end{array}$ \\
\hline No. 1 & $\begin{array}{l}\text { Laceration of the central } \\
\text { part of tendon }\end{array}$ & Standard three-loop pulley & Long lateral splint & 62 & None & 192 \\
\hline No. 2 & $\begin{array}{l}\text { Tendon avulsion from } \\
\text { olecranon }\end{array}$ & Modified three-loop pulley & Long lateral splint & 50 & None & 215 \\
\hline No. 3 & $\begin{array}{l}\text { Tendon avulsion from } \\
\text { olecranon }\end{array}$ & Modified three-loop pulley & Robert-Jones bandage & 49 & $\begin{array}{l}\text { Skin laceration } \\
\text { Avulsion relapse }\end{array}$ & 185 \\
\hline No. 4 & $\begin{array}{l}\text { Tendon avulsion from } \\
\text { olecranon }\end{array}$ & Modified three-loop pulley & Long lateral splint & 81 & None & 243 \\
\hline
\end{tabular}

method using 3-0 nylon. In the 3 cases (Nos. 2-4) with a pattern of tendon rupture at its insertion, the proximal edge of the ruptured tendon formed nodular lesions and was also trimmed using a scalpel (Fig. 3B), and the modified three-loop pulley method was applied to reduce tendon rupture (Fig. 3C). This modified method sutured between bone and tendon, while the standard threeloop pulley sutured between tendon and tendon. A hole was then made in the olecranon transversely using a drill bit; the first loop was passed lateral to medial through the bone tunnel and then medial to lateral through the tendon. After passing through the bone tunnel (lateral-medial) for a second time, the suture material was passed through the tendon at approximately $60^{\circ}$ to the first pass, in a caudomedial-craniolateral direction. Following this, the suture was then passed laterally-medially through the bone tunnel for the final time. The third bite was taken through the tendon at approximately $60^{\circ}$ to the first bite, in a craniomedial-caudolateral direction, and the suture ends were tied. The suture material used in this procedure was $2-0$ or $3-0$ nylon. The incised subcutaneous fat and fascia were sutured using 3-0 to $4-0$ polydioxanone, followed by suturing of the skin using 3-0 to 4-0 nylon.

\section{Postoperative management, complications and outcomes}

External coaptation after surgery was performed in all 4 cases; a long lateral splint was applied to the affected limb in 3 of the cases (Nos. 1, 2, 4), and a Robert-Jones bandage in the remaining case (No. 3). The duration for coaptation was between 49 to 81 days; limited exercise for at least 14 days was instructed in all cases. While there were no postoperative complications reported in the 3 cases (Nos. 1, 2, 4) with long lateral splints, skin laceration occurred around the olecranon in the other case (No. 3) with Robert-jones bandage. Additionally in this case, breakdown of the sutured tendon was confirmed 45 days postoperatively; the operation was repeated. Although the nylon was not broken, the edge of tendon was torn because of the suture material. The threeloop pulley method was applied again after debridement of the injured edge, and a long-leg cylinder cast was applied for external coaptation. The long-leg cylinder cast was removed 63 days after the repeat operation; tendon breakdown did not occur this time. Lameness was not observed at the final clinical examination nor confirmed via telephone conversation with the dog owner in all cases at least 6 months after operation (Table 2).

\section{DISCUSSION}

This study reported the characteristics of triceps brachii tendon injury, which, to the author's knowledge, has only been described in a few cases among cats and dogs $[2,3,5,13,20]$. This is the first report to describe multiple cases of this injury. Although the characteristics of this injury are not widely recognized, the findings of this study indicate that it can be healed through appropriate operative procedures and postoperative management.

This study describes cases of 4 Pomeranians with injured triceps brachii tendon. Although there are no reports discussing the tendon fragility of Pomeranians in comparison with other breeds, variations in the predisposition of different breeds should be considered. A previous study also reported a case of Pomeranian with triceps tendon injury [20]; however, there was no histopathological examination of the triceps brachii. The potential weakness of the triceps brachii tendon in Pomeranians needs to be considered.

The characteristic clinical sign among the all cases was a change in pain level; intense pain developed around humerus and/or elbow joint immediately after injury onset; however, it gradually decreased over time. All 4 dogs could not consciously extend their elbow joint resulting in non-weight bearing posture of the affected limb, because of continued loss of function in the triceps brachii muscle. Additionally the edge of the lacerated tendon formed nodules over several weeks in all cases. This nodular formation has also been described in reports of injury in other tendons $[12,14]$. In this study, the chief complaint of the owners in 2 cases was lameness and nodule formation at the humerus.

The most important examination for identification of an injured triceps brachii tendon was precise palpation. If the tendon break down, the loss of tendon continuity immediately above the olecranon can be detected, especially when elbow joint was flexed. Additionally, nodule formation at the edge of lacerated tendon aided diagnosis in the cases after several weeks from the onset. Lateral projection radiographic images may be useful for visualizing loss of tendon continuity or for detecting bone fragment. While blood examinations, including complete blood count and biochemistry, are not specific, they were useful for acquiring knowledge related to the general condition of the dog and for excluding other diseases. While other imaging tests, such 
as ultrasonography, computerized tomography, and magnetic resonance imaging, were not performed in these cases, they may be valuable tools for improving diagnostic accuracy and for evaluating the postoperative healing of injured tendons [1, 19].

It was very important that any nodule of the lacerated tendon edge was sharply resected using a blade or scissors, until access to grossly normal tendon was achieved. This was performed in order to avoid restricted blood flow across the superficial aspect of the edge, which would have resulted in delayed tendon healing. Many surgical procedures for repairing injured tendons have been reported such as suturing, plating, tendon lengthening, and other methods $[14,17]$. Tendon lengthening was required when the remaining tendon after debridement was too short to connect the edges $[9,14,17]$; however, this procedure was not required in any of the cases reported in this study. The most widely used method for connecting injured tendon is tendon suture. In the previous reports, suture methods were applied to connect the edges of the injured tendons using synthetic absorbable or non-absorbable monofilament material [4]. In this study, the suturing material used in all cases was nylon thread, the size of which (2-0 or 3-0) was determined according to the size of the dog. In the case with a lacerated tendon, the three-loop pulley method was selected for reconstruction. Although many techniques of tendon suture have been proposed, when suturing the edges of tendon, some studies have reported that the three-loop pulley method is stronger than other suturing methods [4, 11]. However, the three-loop pulley method could not be applied in cases of tendon rupture to its insertion because it can only connect tendon to tendon. Therefore, in these cases, the modified three-loop pulley method was applied to the proximal edge of the tendon and the olecranon. This method, first proposed by Moores et al. [11], provided adequate strength.

External coaptation after operation is generally considered essential. The elbow joint should remain extended until complete healing of tendon-tendon or tendon-bone has occurred, because flexing the elbow joint may result in excessive tension in the suturing site. Long lateral splint, or casting is recommended for external coaptation. There were no major complications observed in all 3 cases in which these methods for coaptation were applied; however, in the case in which only a Robert-Jones bandage was used, tendon avulsion was repeated. Skin laceration around the elbow joint occurred in the same case during coaptation; because this is common complication associated with coaptation around the elbow joint, care should be taken during this procedure. According to recommendations from the previous studies of Achilles tendon repair, the duration time of coaptation after surgery was approximately 2 months, and the outcomes were generally excellent, with the exception of the case in which a Robert-Jones bandage was used $[8,14]$.

A major limitation of this study is its retrospective nature; some of the clinical data were not comparable. For example, the method of external coaptation after surgery (long lateral splint and Robert-Jones bandage) depended on surgeon's preference. It is also a limitation that there are no data of some diagnostic imaging such as ultrasonography. At last, improvement of gate is not based on a lameness score or result of force plate, and is just based on a subjective evaluation of the veterinarian and the owner.

In summary, diagnosis of injured triceps brachii tendons can be performed by adequate palpation, and Pomeranians may have a predisposition to this type of injury. If adequate surgery and external coaptation are performed, the prognosis is usually excellent. It is recommend that this injury is considered as a potential diagnosis of non-weight bearing of the forelimb.

\section{REFERENCES}

1. Agnello, K. A., Puchalski, S. M., Wisner, E. R., Schulz, K. S. and Kapatkin, A. S. 2008. Effect of positioning, scan plane, and arthrography on visibility of periarticular canine shoulder soft tissue structures on magnetic resonance images. Vet. Radiol. Ultrasound 49: 529-539. [Medline] [CrossRef]

2. Ambrosius, L., Arnoldy, C., Waller, K. R. 3rd., Little, J. P. and Bleedorn, J. A. 2015. Reconstruction of chronic triceps tendon avulsion using synthetic mesh graft in a dog. Vet. Comp. Orthop. Traumatol. 28: 220-224. [Medline] [CrossRef]

3. Anson, L. W. and Betts, C. W. 2013. Triceps tendon avulsion in a dog: surgical management and xeroradiographic evaluation. J. Am. Anim. Hosp. Assoc. 25: 655-658.

4. Berg, J. R. and Egger, E. L. 1986. In vitro comparison of the three loop pulley and locking loop suture patterns for repair of canine weightbearing tendons and collateral ligaments. Vet. Surg. 15: 107-110. [CrossRef]

5. Davies, J. V. and Clayton Jones, D. G. 1982. Triceps tendon rupture in the dog following corticosteroid injection. J. Small Anim. Pract. 23: 779-787. [CrossRef]

6. Gilmore, D. R. 1984. Triceps tendon avulsion in the dog and cat. J. Am. Anim. Hosp. Assoc. 20: 239-242.

7. Hermanson, J. W. 2013. Muscles of the thoracic limb. pp. 233-254. In: Miller's Anatomy of the Dog 4th ed. (Evans, H. E. and de Lahunta, A. eds.), Elsevier, St. Louis.

8. King, M. and Jerram, R. 2003. Achilles tendon rupture in dogs. Compend. Contin. Educ. Pract. Vet. 25: 613-620.

9. Maffulli, N. 1999. Rupture of the Achilles tendon. J. Bone Joint Surg. Am. 81: 1019-1036. [Medline] [CrossRef]

10. Montgomery, R. and Fitch, R. 2003. Muscle and tendon disorders. pp. 2264-2272. In: Textbook of Small Animal Surgery 3rd ed. (Slatter, D. ed.), Saunders, Philadelphia.

11. Moores, A. P., Comerford, E. J., Tarlton, J. F. and Owen, M. R. 2004. Biomechanical and clinical evaluation of a modified 3-loop pulley suture pattern for reattachment of canine tendons to bone. Vet. Surg. 33: 391-397. [Medline] [CrossRef]

12. Nielsen, C. and Pluhar, G. E. 2006. Outcome following surgical repair of Achilles tendon rupture and comparison between post-operative immobilization methods in dogs 28 cases (1997-2004). Vet. Comp. Orthop. Traumatol. 19: 246-249. [Medline] [CrossRef]

13. Pavletic, M. M., Kalis, R., Tribou, P. and Mouser, P. J. 2015. Triceps brachii muscle reconstruction with a latissimus dorsi muscle flap in a dog. $J$. Am. Vet. Med. Assoc. 246: 226-230. [Medline] [CrossRef]

14. Piermattei, D. L. and Flo, G. L. 2016. Surgical repair of ligaments. pp. 223-226. In: Brinker, Piermattei, and Flo's Handbook of Small Animal Orthopedics and Fracture Repair, 5th ed. (DeCamp, C. E., Johnston, S. A., Dejardin, L. M. and Schaefer, S. L. eds.), Saunders, Philadelphia.

15. Pjanowski, G. J., Stein, L. E. and Turner, T. A. 1989. Strength characteristics and failure modes of suture patterns in severed goat tendons. Vet. Surg. 18: 335-339. [Medline] [CrossRef] 
16. Reinke, J. 1998. Treatment of avulsion of the gastrocnemius tendon. pp. 1257-1260. In: Current Techniques in Small Animal Surgery, 4th ed. (Bojrab, M. J., Ellison, G. W. and Slocum, B. eds.), Williams \& Wilkins, Baltimore.

17. Schulz, K. 2007. Management of muscle and tendon injury or disease. pp. 1316-1332. In: Small Animal Surgery 3rd ed. (Fossum, T. W. ed.), Mosby, St. Louis.

18. Shani, J. and Shahar, R. 2000. Repair of chronic complete traumatic rupture of the common calcaneal tendon in a dog using a fascia lata graft. Vet. Comp. Orthop. Traumatol. 13: 104-108. [CrossRef]

19. Stahl, C., Wacker, C., Weber, U., Forterre, F., Hecht, P., Lang, J. and Gorgas, D. 2010. MRI features of gastrocnemius musculotendinopathy in herding dogs. Vet. Radiol. Ultrasound 51: 380-385. [Medline] [CrossRef]

20. Yoon, H. Y. and Jeong, S. W. 2013. Traumatic triceps tendon avulsion in a dog: magnetic resonance imaging and surgical management evaluation. $J$. Vet. Med. Sci. 75: 1375-1377. [Medline] [CrossRef] 